\title{
„Independence in Europe“? \\ Die Unabhängigkeitsbestrebungen Schottlands nach der Wahl 2011
}

von Stefan Schieren

\begin{abstract}
Nach ihrem historischen Sieg bei den Wahlen zum schottischen Parlament im Mai 2011 erklärte die seither mit absoluter Mehrheit regierende Scottish National Party, bis zum Jahr 2016 die Unabhängigkeit Schottlands erreichen zu wollen. Sie berief sich dabei auf das vermeintliche Recht der Schotten, selbst und alleinig darüber zu bestimmen zu können, in einem souveränen Staat leben zu wollen. Zugleich wird das Versprechen gegeben, dass dieser Staat weiter Mitglied in der EU sein werde. In ihren Bemühungen und mit ihren Versprechen ignoriert die schottische Regierung jedoch die erheblichen rechtlichen und politischen Schwierigkeiten, die es bis zur Unabhängigkeit zu überwinden gilt. So dürfte der Wunsch der Schotten nach Unabhängigkeit, sollte er gesamthaft bestehen, nicht ausreichen, um die „Independence in Europe“ auch tatsächlich zu erlangen. Dazu wird es der stillschweigenden, wahrscheinlich eher der expliziten Zustimmung der europäischen Partner bedürfen. Darauf kann Schottland aber nicht ohne Weiteres zählen.
\end{abstract}

After their victory in the 2011 elections to the Scottish Parliament, the Scottish National Party announced a referendum on Scottish independence due to be held before 2016. The party's claim to "Independence in Europe" rests on an alleged right of the Scottish people to live in a sovereign state according to its own will and choice. However, the matter seems not quite that simple. With regard to international and European law, the Scottish people and the Scottish government will depend on the tacit if not, rather more likely, the explicit understanding of their European partners to gain full independence. Scotland should not, however, take this for granted.

\section{Einleitung}

Die Gründung der Scottish National Party (SNP) im Jahr 1934 als Zusammenschluss von Scottish Party und National Party of Scotland blieb über mehrere Jahrzehnte ohne spürbare Auswirkungen auf die britische oder schottische Politik. Selbst die fulminanten Wahlerfolge in den siebziger Jahren drohten Episode zu bleiben, weil in London nach 1979 eine lange für unbesiegbar gehaltene konservative Partei strikt an der Union und der zentralstaatlichen Organisation des Vereinigten Königreichs festhielt. Die Konservativen sahen und sehen in der als 
„devolution“ bezeichneten Politik der Abgabe autonomer Rechte an Schottland, Wales und Nordirland stets einen ,slippery slope to independence “, 1 den Beginn zum Bruch der Union. ${ }^{2}$ In Schottland wurde diese strikte Ablehnung aber als Missachtung der schottischen Nation interpretiert. Die konservative Partei, nach dem Krieg noch mit Abstand stärkste Kraft, büßte bis 1997 nahezu alle schottischen Wahlkreise ein. Dadurch verstärkten sich das so wahrgenommene „demokratische Defizit" und die Kluft zwischen Edinburgh und London, waren die Schotten doch bis zum Ende des Kabinetts Major weder in der Regierung noch in der Regierungsfraktion vertreten.

Die relative Schwäche der Konservativen begründete die relative Stärke der Labour Partei. Die bittere Wahlniederlage 1992 hatte bei Labour die Überzeugung reifen zu lassen, nur mit Hilfe der schottischen Sitze eine Mehrheit in Westminster gewinnen zu können. ${ }^{3}$ In den siebziger Jahren noch strikte Gegnerin der devolution, ${ }^{4}$ schwenkte sie daher im Laufe der achtziger Jahre auf einen Kurs ein, durch den sich die Partei darauf festlegte, im Falle eines Wahlsiegs die Schotten über die devolution abstimmen lassen zu wollen. ${ }^{5}$ Nach dem Wahlsieg Blairs 1997 votierten beinahe drei Viertel der Wähler in Schottland im September 1998 für ein eigenes schottisches Parlament mit eigener Gesetzgebungshoheit über zahlreiche Politikbereiche (devolved matters). ${ }^{6}$

Nach zwölf Jahren devolution besteht der Anschein, dass die Konservativen mit ihren Bedenken Recht behalten sollen. Seitdem das erste schottische Parlament 1999 gewählt wurde, hat die zu Beginn dominierende Labour Partei von Wahl zu Wahl an Wählerstimmen verloren. Von dieser Entwicklung profitierte nahezu ausschließlich die SNP, die 2007 knapp zur stärksten Fraktion wurde und seither mit einer parlamentarischen Minderheit unter ihrem Parteiführer Alex Salmond regiert.

Obwohl in der Minderheit, kündigte sie in dem Grünbuch „Choosing Scotland's Future“ (August 2007) an, ein Gesetz für ein Referendum über die Unabhängig-

1 Jones, G. W.: Against Regional Government, in: Local Government Studies, 1 - 11, hier: $2 \mathrm{f}$.

2 Major, J.: The Autobiography, London, 1999, 415ff; vgl. Grotz, F., Europäisierung und nationale Staatsorganisation, 2007, 294ff.

3 Vgl. Keating, M.: The Independence of Scotland, Oxford, 2009, 86.

4 Siehe Dalyell, T.: Devolution. The End of Britain?, London, 1977; vgl. Bogdanor, V.: Devolution in the United Kingdom, Oxford, 1999.

5 Vgl. Schieren, S.: Großbritannien, 2. Auflage, Schwalbach im Taunus, 2011, $135 \mathrm{f}$.

6 Vgl. ebd., 137ff; Grotz, F., a.a.O., 2007, 302ff. 
keit durch das Parlament verabschieden lassen zu wollen. ${ }^{7}$ Weil die Mehrheit aus Labour, Liberalen und Konservativen das jedoch ablehnte, musste der First Minister sein Vorhaben zunächst zurückstellen.

Die Wahl im Mai 2011 beseitigte dieses Hindernis. Noch am Wahlabend kündigte der Wahlsieger an, dem schottischen Volk bis 2016 ein Referendum über die Loslösung vom United Kingdom of Great Britain and Northern Ireland vorlegen zu wollen. ${ }^{8}$ Durch diese Wahl ist die SNP ihrem Ziel, der Gründung eines souveränen Schottlands, näher gekommen als je zuvor in ihrer Geschichte.

Tabelle 1: Wahlergebnisse 1999 bis 2007

\begin{tabular}{l|c|c|c|c|c|c}
\hline \multirow{2}{*}{ Partei } & \multicolumn{2}{|c|}{ Wahlergebnis 1999} & \multicolumn{2}{c|}{ Wahlergebnis 2003} & \multicolumn{2}{c}{ Wahlergebnis 2007} \\
& Wahlkreis & Region & Wahlkreis & Region & Wahlkreis & Region \\
\hline SNP & $28.7 \%$ & $27.3 \%$ & $23.8 \%$ & $20.9 \%$ & $32.9 \%$ & $31.0 \%$ \\
\hline Labour & $38.8 \%$ & $33.6 \%$ & $34.6 \%$ & $29.3 \%$ & $32.1 \%$ & $29.2 \%$ \\
\hline Konserv. & $15.6 \%$ & $15.4 \%$ & $16.6 \%$ & $15.5 \%$ & $16.6 \%$ & $13.9 \%$ \\
\hline LibDem & $14.2 \%$ & $12.4 \%$ & $15.4 \%$ & $11.8 \%$ & $16.2 \%$ & $11.3 \%$ \\
\hline Sonst. & $0.0 \%$ & $3.6 \%$ & $0.0 \%$ & $6.9 \%$ & $0.1 \%$ & $4.0 \%$ \\
\hline
\end{tabular}

Quelle: Schieren, S., a.a.O., 2011: 140.

Tabelle 2: Wahlergebnis 2011

\begin{tabular}{l|c|c|c|c}
\hline Partei & Sitze absolut & Anteil der Sitze & $\begin{array}{c}\text { Wahlkreis- } \\
\text { stimmen }\end{array}$ & Regionalstimmen \\
\hline SNP & 69 & $53.5 \%$ & $45.4 \%$ & $44.0 \%$ \\
\hline Labour & 37 & $28.7 \%$ & $31.7 \%$ & $26.3 \%$ \\
\hline Konserv. & 15 & $11.6 \%$ & $13.9 \%$ & $12.4 \%$ \\
\hline LibDem & 5 & $3.9 \%$ & $7.9 \%$ & $5.2 \%$ \\
\hline Green & 2 & $1.6 \%$ & - & $4.4 \%$ \\
\hline M. MacD. & 1 & $0.8 \%$ & - & $0.9 \%$ \\
\hline Sonstige & - & - & $1.1 \%$ & $6.8 \%$ \\
\hline
\end{tabular}

Quelle: Herbert, S. u.a.: Election 2011. Scottish Parliament Information Centre (SPICe), Edinburgh, 2011, 3 .

7 Scottish Government: Choosing Scotland's Future, Edinburg, 2007, V.

8 Vgl. „Scottish independence: Cameron gives green light to referendum“, in: The Guardian online, 08.05.2011; ,Scottish independence referendum“, in: BBC News Scotland online, 29.05.2011. 
Doch wie nahe ist sie diesem Ziel tatsächlich gekommen? Wie verhält es sich mit einer Abspaltung von Großbritannien? ${ }^{9}$ Haben die Schotten ein Recht zur Sezession? ${ }^{10}$ Können sie sich dazu auf das Selbstbestimmungsrecht der Völker berufen? Was würde aus einer Sezession mit Blick auf die Mitgliedschaft in der EU folgen? Welchen Gehalt hat der SNP Wahlslogan „Independence in Europe“, mit dem die SNP zaudernden Schotten die Angst zu nehmen versucht, am Rande Europas nicht überlebensfähig zu sein? Kann die SNP dieses Wahlversprechen überhaupt einlösen? Mit anderen Worten: Könnte ein souveräner Staat Schottland für sich das Recht beanspruchen, ohne Unterbrechung Mitglied in der EU $\mathrm{zu}$ bleiben, oder ist es diesbezüglich auf die Zustimmung Großbritanniens und/oder der anderen Mitgliedstaaten angewiesen?

Die schottische Regierung scheint jedenfalls davon auszugehen, dass die Mitgliedschaft in der EU außer Frage steht. „An independent Scotland would continue in the European Union and bear the burdens and fulfil the responsibilities of membership" heißt es im benannten Grünbuch der schottischen Regierung lapidar. Mehr noch, die Regierung scheint sich sogar in der Position zu wähnen, über die Konditionen der Mitgliedschaft Verhandlungen führen zu können. ${ }^{11}$

\section{Das Selbstbestimmungsrecht der Völker}

\section{Grundlagen}

In ihren Forderungen nach Unabhängigkeit haben die Vertreter der SNP immer wieder auf das im Völkerrecht verankerte Selbstbestimmungsrecht der Völker als Grundlage für ihren Unabhängigkeitsanspruch verwiesen. ${ }^{12}$ Die zunächst zu klärende Frage lautet folglich, ob Schottland bzw. die Schotten aus dem Selbst-

9 Eine Reihe von Überlegungen, die die schottische Regierung außer Acht lässt, ist bereits von Attwooll angestellt worden (Attwooll, E.: A Right to Secede? Scotland Reviewed, in: Bellamy, R. (Hg.): Constitutionalism, Democracy and Sovereignty. American and European Perspectives, Aldershot u.a., 1996, 133 - 150. Seither hat sich allerdings eine Fülle neuer Aspekte ergeben, die eine neuerliche Betrachtung berechtigt erscheinen lassen.

10 Vgl. „Scottish independence: Cameron gives green light to referendum.“, in: The Guardian online, 08.05.2011.

11 Vgl. Hazell, R.: Salmond has far to leap. The Scottish Route to Independence is More Complicated then the SNP would have you believe, in: Prospect, Juli 2008, 18 - 19, hier 18.

12 So lässt sich die Position der schottischen Regierung verstehen, das schottische Volk besitze das ,right to determine their own constitutional position, whether they choose that of an independent sovereign state, or that of membership of the United Kingdom as at present". Scottish Government, a.a.O., 2007, 20. 
bestimmungsrecht der Völker das Recht auf staatliche Unabhängigkeit ableiten können.

Seit der amerikanische Präsident Woodrow Wilson in seinen 14 Punkten der Idee eines Selbstbestimmungsrechts der Völker zum Durchbruch verhalf, hat sie die internationale Politik stark beeinflusst. ${ }^{13}$ Jörg Fisch wies in seiner Betrachtung dieser Idee auf die Widersprüchlichkeiten und auch auf die unheilvollen Folgen dieser „Illusion“ hin. ${ }^{14}$ Ungeachtet der beileibe nicht nur günstigen historischen Erfahrungen ist das Selbstbestimmungsrecht der Völker dennoch nicht mehr aus dem Völkerrecht wegzudenken. Es fand Niederschlag in vielen Dokumenten ${ }^{15}$ und trägt inzwischen - so die herrschende Meinung der Völkerrechtslehre sogar den Status eines ius cogens. Es ist ein Recht, dass der Rechtsträger zu beanspruchen nicht alleine berechtigt ist, sondern dass die anderen Völkerrechtssubjekte verpflichtet sind, notfalls in dessen Interesse durchzusetzen. ${ }^{16}$

\section{Träger des Selbstbestimmungsrechts}

Der Befund ist folglich, dass es ein Selbstbestimmungsrecht der Völker gibt. Doch was daraus folgt, ist unklar und umstritten. Wer ist Träger dieses Rechts, und wie verhält es sich mit dem Recht auf Sezession vom Mutterland?

Die erste Frage mutet prima facie etwas uninspiriert an, scheint der Träger des Rechts - das Volk - doch bereits im Begriff selbst genannt zu sein. Allein: Der Begriff „Volk“ ist alles andere als klar ${ }^{17}$ und lädt zu Missverständnissen ein. Er hält für gänzlich gegensätzliche politische Ziele passend erscheinende Argumente parat. „Damit wird erneut die zentrale Schwierigkeit sichtbar, mit der die

13 Vgl. Thürer, D.: Das Selbstbestimmungsrecht der Völker und die Anerkennung neuer Staaten, in: Neuhold, H./Simma, B. (Hg.): Neues europäisches Völkerrecht nach dem Ende des Ost-WestKonfliktes?, Baden-Baden, 1996, 43 - 58, hier 43.

14 Fisch, J.: Das Selbstbestimmungsrecht der Völker, München, 2010. Vgl. auch Koskenniemi, M.: National Self-Determination Today. Problems of Legal Theory and Practice, in: McCorquendale, R. (Hg.): Self-Determination in International Law, Aldershot, 2000, 555 - 583.

15 Das bezieht sich besonders auf die Friendly Relations Resolution, General Assembly 2625 (XXV), in: UNYB 1970, 788. Siehe auch Decolonization Resolution, General Assembly 1514 (XV), in: UNYB 1960 49. Vgl. Thürer, D., a.a.O., 48ff.

16 Ott, M.: Das Recht der Sezession als Ausfluss des Selbstbestimmungsrechts der Völker, Berlin, 2008, 74ff; Tomuschat, Chr.: Secession and Self-Determination, in: Kohen, M. G. (Hg.): Secession. International Law Perspectives. Cambridge, 2006, 23 - 45, hier 38ff.

17 Vgl. Murswiek, D.: Offensives und defensives Selbstbestimmungsrecht. Zum Subjekt des Selbstbestimmungsrechts der Völker, in: Der Staat 23 (1984), 523 - 548. 
Selbstbestimmungsrechtsformel verbunden ist. Sie enthält ein Versprechen, das nicht eingelöst werden kann“. ${ }^{18}$

Wer ist Träger des Rechts? Die Staaten als politischer Körper eines Volkes? In diesem Fall dient es dem Zweck, die staatliche Souveränität und territoriale Integrität eines Staates zu behaupten. Diese Auffassung lässt sich zum Beispiel der UN-Charta entnehmen, ${ }^{19}$ die das Prinzip der territorialen Integrität betont. Diese Auffassung betrachtet in der Abspaltung vom Mutterland nicht die Verwirklichung des Selbstbestimmungsrechts, sondern einen Verstoß dagegen. ${ }^{20}$

Die objektive Theorie ${ }^{21}$ hingegen spricht von einem „Volk“, wenn es durch Gemeinsamkeiten wie Sprache, Religion, Abstammung, Geschichte und eine ,,ausgeprägte kollektive Identität" gekennzeichnet ist und die diese Merkmale tragenden Menschen in einem geschlossenen Territorium leben. ${ }^{22}$ Eine Mindermeinung im Völkerrecht ${ }^{23}$ schlussfolgert daraus, dass das Selbstbestimmungsrecht nur in einem unabhängigen Staat zu gewährleisten sei. Dessen Verwirklichung wird also direkt an das Recht zur Sezession geknüpft.

\section{Ein Recht auf Sezession?}

\section{Selbstbestimmungsrecht und Menschenrechte}

In der Völkerrechtslehre hat sich eine Zwischenposition etabliert. Das Selbstbestimmungsrecht wird nicht gleichbedeutend mit der Bewahrung der territorialen Identität verstanden, aus ihm wird aber auch nicht der unmittelbare Anspruch auf Abspaltung abgeleitet, wie es sich im Zuge der Entkolonialisierung anzudeuten schien.

Verantwortlich dafür sind die Erfahrungen beim Dekolonisationsprozess. Freiheitskämpfer in vielen Kolonien hatten unter Berufung auf das Selbstbestimmungsrecht die Herrschaft der Kolonialmacht abgeschüttelt, um im Gegenzug

18 Fisch, J., a.a.O., 2010, 23.

19 Vgl. Ott, M., a.a.O., 2008, $104 \mathrm{ff}$.

20 Vgl. Hannum, H.: Autonomy, Sovereignty, and Self-Determination, Philadelphia, 1996, 453f.

21 Der subjektiven Theorie zufolge reicht es, wenn eine Großgruppe sich selbst zum „Volk“ erklärt. Vgl. etwa Beran, H.: A Liberal Theory of Secession, in: Political Studies 32 (1984), 21 - 31. Diese Auffassung hat sich nicht durchgesetzt. Vgl. Schneckener, U.: Leviathan im Zerfall. Über Selbstbestimmung und Sezession, in: Leviathan 25 (1997), 458 - 479; Ott, M., a.a.O., 113.

22 Ott, M., a.a.O., 2008, $114 \mathrm{ff}$.

23 Vgl. ebd., 425f. 
Gewaltregime zu errichten, die schreckliche Verbrechen an ihren Bürgern verübten. ${ }^{24}$ Idi Amin ist zur Chiffre für diese Katastrophen geworden.

Vor dem Hintergrund dieser ernüchternden Erfahrungen wurde in der Völkerrechtslehre die Auffassung entwickelt, dass die nach 1945 entstandenen Dokumente zum Selbstbestimmungsrecht exklusiv auf den Dekolonisationsprozess anzuwenden seien und ihnen keine generelle Geltung zukomme. Hätte sich diese Auffassung durchgesetzt, wäre die Bedeutung des Selbstbestimmungsrechts wieder maßgeblich auf die Forderung nach territorialer Integrität verengt worden. ${ }^{25}$ Sie blieb zwar Mindermeinung, ${ }^{26}$ ist aber Beleg dafür, dass dieser Aspekt gesteigerte Bedeutung gewann, und dass ein Recht auf Sezession zunehmend zurückhaltend betrachtet wurde.

Ferner wurde die Idee vom Selbstbestimmungsrecht im letzten Drittel des 20. Jahrhunderts mit anderen politischen Ideen verknüpft. Im gewandelten Verständnis hob es weniger auf die Rechte eines Volkes als Kollektiv ab, als auf die den Mitgliedern eines Volkes zukommenden individuellen Rechte wie Menschenrechte, Recht auf Selbstregierung und Recht auf gutes Regieren. Das Selbstbestimmungsrecht der Völker sollte nicht länger dafür missbraucht werden können, korrupte und gierige Gewaltregime zu errichten. „Das Selbstbestimmungsrecht ist ... kein Menschenrecht, sondern wesentliche Voraussetzung für den Genuss von Individualrechten. Der Menschenrechtsschutz ist seinerseits maßgebliche Bedingung für die Selbstbestimmung der Völker“. ${ }^{27}$

In dieser gewandelten Lesart folgt das Recht auf staatliche Unabhängigkeit nur unter sehr rigiden Bedingungen aus dem Selbstbestimmungsrecht der Völker. Es kann nur als ,a self-helping remedy in causes of extreme oppression ${ }^{\text {“28 }}$ aktiviert werden. Solange keine extreme Unterdrückung mit schweren Menschenrechtsverletzungen vorliegt, rangiert die Wahrung der territorialen Integrität vor dem Recht auf Sezession. ${ }^{29}$

24 Vgl. Fisch, J., a.a.O., 2010, $217 \mathrm{ff}$.

25 Simpson, G. J.: The Diffusion of Sovereignty. Self-determination in the Post-Colonial Age, in: Sellers, M. N. (Hg.): The New World Order. Sovereignty, Human Rights, and Self-Determination of Peoples, Oxford, 1996, 37 - 69, hier 40ff; Ott, M., a.a.O., 2008, 76.

26 Vgl. Ott, M., a.a.O., 2008, 76.

$27 \mathrm{Vgl}$. ebd., 82f.

28 So Buchheits einflussreiche Bestimmung; Buchheit, L. C.: Secession. The Legitimacy of Selfdetermination, Princeton, 1978, 64f); vgl. Ott, M., a.a.O., 2008, 444; Tomuschat, Chr., a.a.O., 2006, $34 \mathrm{ff}$.

29 Vgl. Ott, M., a.a.O., 2008, 425; vgl. ebd. 438; 444ff; Cismas, I.: Secession in Theory and Practice. The Case of Kosovo and Beyond, in: Goettingen Journal of International Law 2 (2010), 531 - 587, hier 549f. 


\section{Scottish independence nach der devolution 1999}

Es ist offenkundig, dass diese Voraussetzungen in Schottland nicht erfüllt sind. Auch wenn die Umstände, unter denen Schottland im 18. Jahrhundert zu einem integralen Bestandteil des Vereinigten Königreichs wurde, gewiss hinterfragt werden können, weil massive Bestechung im Spiel war, sind die Beziehungen zwischen Schottland und England seit dem 19. Jahrhundert dadurch geprägt, dass es einen fortlaufenden und beschleunigten Transfer von autonomen Rechten nach Edinburgh gab. ${ }^{30}$ Dieser Prozess gipfelte 1997 in den Referenden über die devolution und 1999 in der Einberufung eines eigenen Parlaments.

Ferner ist zu bedenken, dass die öffentlichen Ausgaben in Schottland im Vergleich zum gesamten Vereinigten Königreich überdurchschnittlich hoch sind. ${ }^{31}$ Auch das Pro-Kopf-Einkommen liegt mittlerweile um $12 \%$ über dem in England. ${ }^{32}$ Die Möglichkeit, sich unter Berufung auf das Selbstbestimmungsrecht der Völker von der Union mit England loszusagen, war zu keinem Zeitpunkt weniger naheliegend als gegenwärtig.

\section{Scottish independence und die KSZE}

Nach dem Zerfall des Ostblocks 1989/91 sind zahlreiche Staaten in Europa verschwunden bzw. neu entstanden, einige nach einer einseitigen und gewaltsam erzwungenen Sezession. Die Frage ist, ob in Folge dieser politischen Entwicklung in Europa ein Rechtszustand erwuchs, der eine für Schottlands Unabhängigkeitsbestrebungen günstigere Ausgangsposition darstellt, als sie das Völkerrecht bietet. ${ }^{33}$

Die KSZE-Schlussakte bekräftigt das Recht auf Selbstbestimmung. Doch kommt dem aus zwei Gründen keine weitere Bedeutung zu. Zum einen ist die KSZE

30 Münter, M.: Verfassungsreform im Einheitsstaat. Die Politik der Zentralisierung in Großbritannien, Wiesbaden, 2005, 63 - 98; Grotz, F., a.a.O., 2007, 272 - 279.

31 Hazell, R.: Introduction. What is the English Question?, in: ders. (Hg): The English Question, Manchester/New York, 2006, 1 - 21, hier 9.

32 "Does Scotland have too much to lose from independence?", in: BBC News UK online, 04.07.2011. . 2001 lag das BIP noch 4 \% unter dem Durchschnitt des Vereinigten Königreichs, vgl. Trench, A.: Scotland and Wales. The Evolution of Devolution, in: Hazell, R. (Hg.): Constitutional Futures Revisted. Britain's Constitution to 2020, London, 2008, 35.

33 Oeter weist auf die Auswirkungen der Jugoslawienkrise auf das Recht und die Praxis der internationalen Beziehungen hin. Oeter, St:: Völkerrechtliche Rahmenbedingungen und die Staatengemeinschaft, in: Melčić, D. (Hg.): Der Jugoslawien-Krieg, Opladen/Wiesbaden, 1999, 478 - 498. 
kein Völkerrechtssubjekt. Es handelt sich bei der Schlussakte nicht um ein Rechtsdokument, sondern um die Bekräftigung eines Prinzips. ${ }^{34}$

Zum anderen hat sich im weiteren Verlauf des Konsultationsprozesses gezeigt, dass die Europäer unter dem Selbstbestimmungsrecht der Völker die innerstaatliche Garantie der Menschenrechte und die Stärkung der Selbstregierung von Minderheiten verstehen. Deren Gewährleistung sollte den Wunsch und das Recht auf Sezession erst gar nicht entstehen lassen. Dieses Verständnis folgt dem Gedanken nach territorialer Integrität, indem es ein Recht auf Sezession zurückweist.

Die „Charta von Paris für ein neues Europa“635 vom November 1990 erneuert als Grundlage für die ,,freundschaftliche[n] Beziehungen zwischen den Teilnehmerstaaten“ das „feierliche Versprechen, uns jeder gegen die territoriale Integrität oder politische Unabhängigkeit eines Staates gerichteten Androhung oder Anwendung von Gewalt oder jeder sonstigen mit den Grundsätzen oder Zielen dieser Dokumente unvereinbaren Handlung zu enthalten ... Wir bekräftigen die Gleichberechtigung der Völker und ihr Selbstbestimmungsrecht in Übereinstimmung mit der Charta der Vereinten Nationen und den einschlägigen Normen des Völkerrechts, einschließlich jener, die sich auf die territoriale Integrität der Staaten beziehen."

Die Schlusserklärung der Konferenz über die „Human Dimension of the CSCE ${ }^{\text {‘36 }}$ im Oktober 1991 in Moskau machte im Licht der heraufziehenden Jugoslawienkrise sogar deutlich, dem Grundsatz der Unverletzlichkeit der territorialen Integrität sei ein höheres Gewicht beizumessen als dem Recht auf staatliche Unabhängigkeit. Damit wurde auch die Haltung der europäischen Staaten in der Jugoslawien-Krise abgesteckt. Die Anerkennung neuer Staaten wurde nur als „letzter Ausweg“ akzeptiert. ${ }^{37}$ Die KSZE erzeugt mithin keine Rechtsgrundlage für etwaige schottische Ansprüche. ${ }^{38}$

34 Vgl. Pazartzis, Ph.: Secession and International Law. The European Dimension, in: Kohen, M. G. (Hg.): Secession. International Law Perspectives. Cambridge 2006, 355 - 373, hier 358.

35 Presse- und Informationsamt der Bundesregierung: Bulletin Nr. 137, 24. November 1990, 1409-1415.

36 Europa-Archiv 46 (1991) D 579.

37 Vgl. Rich, R.: Recognition of States. The Collapse of Yugoslavia and the Soviet Union, in: European Journal of International Law 4 (1993), 36 - 65, hier 42f.

38 Ott, M., a.a.O., 2008, 70 f. 


\section{Scottish independence und die Zeitenwende 1989/91}

Diese Bekräftigung des Prinzips der territorialen Integrität durch die KSZE und die europäischen Staaten in der Zeitenwende 1989/91 erfolgte nicht zuletzt vor dem Hintergrund der Befürchtung, dass der Zerfall des Ostblocks schon schwer genug zu beherrschen sein würde. Die Betonung eines Selbstbestimmungsrechts der Völker hätte als eine unpassende Ermutigung der Unabhängigkeitsbestrebungen in vielen Ostblockstaaten verstanden werden können, die die ohnehin angespannte Situation nur weiter verschärft hätte.

Am Ende erwies sich der Zerfall der Sowjetunion, der Tschechoslowakei und Jugoslawiens als unaufhaltbar. Das Zerbrechen ${ }^{39}$ der Tschechoslowakei in Tschechien und in die Slowakei verlief ungeachtet erheblicher Spannungen und Meinungsunterschiede im Vorfeld letztlich einvernehmlich, friedlich und völkerrechtskonform. ${ }^{40}$ Beide Teile versicherten sich gegenseitig des Rechts zur Nachfolge in die von der ČSSR vereinbarten internationalen Verträge. ${ }^{41}$

Die Sowjetunion versuchte hingegen bis zum Beginn ihres Zerfalls nach dem Putsch im August 1991, die Unabhängigkeit ihrer Republiken auch gewaltsam zu verhindern. Das blieb jedoch eine - für die Betroffenen schreckliche - Episode. Vor dem Hintergrund dieser Ereignisse erfolgte die Entstehung von 16 neuen Staaten aus dem „Nachlass“ der UdSSR doch insgesamt - von Ausnahmen abgesehen - ohne gewaltigere Erschütterungen. ${ }^{42}$

Nach anfänglich vergleichbarer Entwicklung verhielt es sich mit dem Zerfall Jugoslawiens bald gänzlich anders. Ende 1991 kam es zu einem Zusammenbruch der staatlichen Autorität und zum Ausbruch nackter Gewalt. Unter dem Druck Deutschlands rangen sich die europäischen Staaten gegen schwere Bedenken zur Anerkennung neuer Staaten auf dem Territorium des ehemaligen Jugoslawiens durch. Dabei machten die Staaten unmissverständlich deutlich, dass die Situation

39 Die Sezession ist die einvernehmliche oder auch einseitige Abspaltung eines Territoriums von einem Staat, der einen neuen Staat bildet. Die „Dismembration“ ist der Zerfall eines Staates in mehrere Bestandteile, ohne dass einer der neuen Staaten als Nachfolgestaat des untergegangenen Staates anerkannt werden würde. Vgl. Ott, M., a.a.O., 47.

40 Vgl. Hertig, M.: Die Auflösung der Tschechoslowakei. Analyse einer friedlichen Staatsteilung, Basel u.a., 2001, $173 \mathrm{ff} ; 279 \mathrm{ff}$.

41 Vgl. Schweisfurth, T.: Das Recht der Staatensukzession. Die Staatenpraxis der Nachfolge in völkerrechtliche Verträge, Staatsvermögen, Staatsschulden und Archive in den Teilungsfällen Sowjetunion, Tschechoslowakei und Jugoslawien, in: Das Recht der Staatensukzession, Berichte der Deutschen Gesellschaft für Völkerrecht, Bd. 35, Heidelberg, 1995, 43 - 125, hier 82ff.

42 Vgl. ebd., 81f. 
in Jugoslawien keinen anderen Ausweg ließ. ${ }^{43}$ Außerdem stellten sie fest, dass es zum Zerfall Jugoslawiens (,dismembration“) gekommen sei, nicht etwa zur Abspaltung eines Territoriums bei Fortbestehen eines Vorgängerstaats. ${ }^{44}$ Und schließlich wurde der bisher dem Dekolonisationsprozess vorbehaltene uti possidetis-Grundsatz als Richtschnur für Europa formuliert. ${ }^{45}$ Eine Teilung als letztes Mittel darf nur entlang bestehender innerstaatlicher Grenzen erfolgen. Das alles geschah in dem Interesse, der Wahrung des territorialen Prinzips den Vorrang vor dem Recht auf Sezession zu sichern. Die Sezession sollte extreme Ausnahme bleiben. Schon gar nicht sollte sie bedingungslos aus dem Selbstbestimmungsrecht der Völker abgeleitet werden können. ${ }^{46}$

Im Kosovo-Konflikt sollte sich erweisen, wie stark die Vorbehalte der EU gegen die Anerkennung von weiteren Staaten waren. Lange Zeit zog sie den Status des UN-Protektorats einer Anerkennung der Unabhängigkeit vor. Als sich die einseitige Unabhängigkeitserklärung des Kosovo 2008 nicht mehr verhindern ließ, erklärten die Außenminister der EU bzw. der USA schleunigst, es handle sich um einen ,sui generis case“ bzw. einem „special case“, der keinen ,precedent for any other situation in the world today" darstelle. ${ }^{47}$ Die Bewahrung der territorialen Integrität sollte ihren herausragenden Stellenwert erhalten.

\section{Zwischenergebnis}

Das Selbstbestimmungsrecht der Völker ist als allgemein anerkannter Grundsatz des Völkerrechts auf Schottland anwendbar. Die Schotten sind ein Volk und unzweifelhaft Träger des Selbstbestimmungsrechts. Daraus folgen aber nicht das Recht auf einen eigenen, souveränen Staat und damit auch nicht ein rechtlich verbriefter Anspruch auf Abspaltung.

43 Vgl. Tancredi, A.: A Normative 'Due Process' in the Creation of States through Secession, in: Kohen, M. G. (Hg.): Secession, Cambridge, 2006, 171 - 207, hier $185 f$.

44 Vgl. Schweisfurth, T., a.a.O., 1995, 56ff.

45 Vgl. Oeter, S., a.a.O., 1999, 481ff. Die EG-Staaten hatten im August 1991 die so gennannte BadinterKommission eingesetzt, die maßgebliche Vorarbeiten für die Beurteilung der Situation in Jugoslawien für die EG lieferte. Den Vorsitz der Kommission hatte der derzeitige Präsident des französischen Verfassungsgerichts, Robert Badinter. Ihr gehörten ferner die Präsidenten der Verfassungsgerichte von Deutschland, Italien, Spanien und Belgien an. Vgl. auch Rich, R., a.a.O., 1993, 48.

46 Dementsprechend hieß es im 2. Badinter-Gutachten, das Selbstbestimmungsrecht ,must not involve changes to the existing frontiers". Abgedruckt in European Journal of International Law 3 (1992), S. 182 - 185. Vgl. Tomuschat, Chr., a.a.O., 2006, 37f; Dugard, J./Raič, D.: The Role of Recognition in the Law and Practice of Secession, in: Kohen, M. G. (Hg.): Secession, Cambridge 2006, 94 - 137, hier 134; Tancredi, A., a.a.O., 2006, $187 \mathrm{f}$.

47 Zit. nach Cismas, I., a.a.O., 2010, 584f. 
In einem Schottland ähnlich gelagerten Fall hatte der kanadische Oberste Gerichtshof im Fall von Quebec nicht erkennen können, dass der Bundesstaat Kanada dem Gliedstaat Quebec das Selbstbestimmungsrecht versage, weswegen er weder auf der Basis der kanadischen Verfassung noch auf der des internationalen Rechts eine Grundlage für eine einseitige Sezession sah. Quebec wurde lediglich zugestanden, dass es im Falle einer sich für die Sezession aussprechenden Volksabstimmung Anspruch auf Verhandlungen mit der kanadischen Zentralregierung besitze. $^{48}$

Der Abfall von der Sowjetunion erfolgte in Folge des Zusammenbruchs der inneren Ordnung. Ferner bleibt zu bedenken, dass die Verfassung der UdSSR den Teilrepubliken das Recht zum Austritt aus der Union zugestand. ${ }^{49}$ Einen derartigen Anspruch enthält die britische Verfassung nicht.

Beim Zerfall Jugoslawiens ist ebenfalls zu beachten, dass der Bundesstaat seinen Gliedstaaten den Austritt erlaubte. ${ }^{50}$ Dennoch zeigten die europäischen Staaten starke Vorbehalte gegen den Zerfall und ließen erkennen, dass sie nur in sehr engen Grenzen bereit seien, eine Sezession zu akzeptieren.

Stehen die Dinge somit nach internationalem und europäischem Recht nicht günstig, ist im Folgenden zu betrachten, wie sich das britische Recht zu einer Auflösung der Union verhält; international liefert allenfalls die Tschechoslowakei Stoff für einen Präzedenzfall. Die Teilung des Landes erfolgte einvernehmlich. Dieses Einvernehmen war die Voraussetzung dafür, dass es hinsichtlich der internationalen Anerkennung keine Probleme gab, besonders mit Blick auf die Rechtsnachfolge bei Staatsverträgen, obwohl es eine Reihe heikler Fragen zu bedenken galt. ${ }^{51}$ An diesem Maßstab wird Schottland sich messen lassen müssen. Es darf als sicher gelten, dass Schottland sich als souveräner Staat nur dann etablieren kann, wenn es zu einer rechtmäßigen Sezession kommt. Denn davon wird die Anerkennung durch die anderen europäischen Staaten abhängen. ${ }^{52} \mathrm{Wie}$ die Bedingungen dafür stehen, wird im Folgenden betrachtet.

48 Vgl. Keating, M, a.a.O., 2009, 82; Ott, M., a.a.O., 2008, 404ff; Dumberry, P.: Lessons learned from the Quebec Secession Reference before the Supreme Court of Canada, in: Kohen, M. G. (Hg.): Secession, Cambridge, 2006, 416 - 452, hier 422ff.

49 Art. 72 Verfassung der UdSSR: Jeder Unionsrepublik bleibt das Recht auf freien Austritt aus der UdSSR gewahrt.

50 Vgl. Cismas, I., a.a.O., 2010, 543.

51 Vgl. Hertig, M., a.a.O., 2001, $390 \mathrm{ff}$.

52 Vgl. Dugard, J./Raič, D., a.a.O., 2006, 135. 


\section{Die Abspaltung Schottlands im britischen Recht}

\section{Ausgangslage}

Auf die Ankündigung eines Unabhängigkeitsreferendums erklärte Premierminister David Cameron unverzüglich, dass er und seine Regierung ihm weder rechtliche noch politische Hürden in den Weg legen wollten, auch wenn seine Rechtmäßigkeit in Frage stehe. ${ }^{53}$ Gleichzeitig kündigte er an, leidenschaftlich gegen die Sezession und für den Erhalt der Union kämpfen zu wollen.

Der Regierungschef erhielt darin Unterstützung von zahlreichen Politikern aus Regierung und auch seitens der Opposition, ${ }^{54}$ wäre für diese der Wegfall der schottischen Wahlkreise doch eine schwere Hypothek für die Mehrheitsfähigkeit in Westminster.

Umfragen in England zeigen zudem eine starke Abneigung gegen eine Abspaltung Schottlands, verknüpft mit der Forderung, im Falle des Falles auch in England eine Volksabstimmung über die Sezession abzuhalten zu wollen. ${ }^{55}$

$\mathrm{Ob}$ und wie lange der Premier vor diesem Hintergrund zu seiner ursprünglichen Aussage wird stehen können, zumal dann, wenn in der eigenen, traditionell unionistischen Partei die Widerstände wachsen, ist nicht gewiss. ${ }^{56}$ Die innerparteiliche Abwehr dürfte zudem weiter steigen, wenn es um eine Einigung über die reichen Erdölvorkommen in der Nordsee gehen wird. ${ }^{57}$

Sollte es zu einem Meinungswechsel kommen, wie beispielsweise vor einigen Jahren in der Frage einer Volksabstimmung über den Euro-Beitritt des Landes, stünden London die rechtlichen Instrumente zur Verfügung, ein Unabhängigkeitsreferendum in Schottland $\mathrm{zu}$ verhindern. Die politischen Institutionen Schottlands verfügen nicht über die Kompetenz, über Verfassungsfragen zu befinden. Diese Kompetenz liegt ausschließlich beim Parlament in Westminster. Würden das Parlament und die Regierung in Edinburgh dies ignorieren, hätte der

53 Vgl. „Scottish independence: Cameron gives green light to referendum. Prime minister tells Alex Salmond he will not put up any legal or political objections to a vote“, The Guardian online, 08.05.2011.

54 „Alistair Darling vows to fight against independence“, in: BBC News Scotland online, 09.07.2011. .

55 „Nearly half of English oppose Scottish independence-poll“, in: BBC News Scotland online, 04.07.2011. Ältere Erhebungen ergeben allerdings ein weniger klares Bild. Hazell, R., Introduction, a.a.O., 2008, 7f; Aughey, A.: The Future of Britishness, in: Hazell, R. (Hg.): Constitutional Futures Revisted. Britain's Constitution to 2020, London, 2008, 90 - 105, hier 98.

56 Vgl. "Scottish nationalists angry over double referendum hurdle", in: The Guardian online, 06.06.2011..

57 „Does Scotland have too much to lose from independence?“, in: BBC News UK online, 04.07.2011. ; Hazell, R., Salmond, 2008, 18f. 
für „,devolution matters“ zuständige ${ }^{58}$ Supreme Court kaum eine andere Möglichkeit, als einen Rechtsbruch festzustellen. ${ }^{59}$ Eine rechtlich saubere Lösung bestünde ausschließlich darin, dass das Westminster Parlament durch eigenes Gesetz das Referendum ermöglicht oder das schottische Parlament unter Sec. 30 (2) Scottish Act 1998 ermächtigt, ein entsprechendes Gesetz zu erlassen.

Weil die Schotten aber kein Interesse daran haben können, gleich beim ersten Schritt in die Unabhängigkeit einen Rechtsbruch zu begehen, wurde der Vorschlag gemacht, ein Referendum nicht direkt über die Frage der Unabhängigkeit abzuhalten, ${ }^{60}$ sondern die schottische Regierung durch Volksabstimmung zu ermächtigen, Verhandlungen mit London über die Unabhängigkeit Schottlands aufzunehmen. Das politische Kalkül dahinter ist, einen positiven Ausgang des Referendums gleichzusetzen mit einer Entscheidung über die Unabhängigkeit selbst. $^{61}$

Unzweifelhaft könnte eine Regierung in Whitehall den positiven Ausgang einer Volksabstimmung nicht ignorieren, in der sich die Schotten für die Unabhängigkeit aussprechen. Ein Referendum über die Ermächtigung der Regierung wäre jedoch noch ein gutes Stück von einem solchen Votum entfernt. London verfügte in solchen Verhandlungen über günstige Ausgangsbedingungen, da Schottland mit Blick auf die Beziehungen sowohl zu England als auch zur EU auf das Einvernehmen seiner Partner angewiesen wäre. Beide können den Schotten die Unabhängigkeit so unattraktiv machen, dass diese sich mit Blick auf das Verhandlungsergebnis schließlich doch noch gegen diesen Schritt entscheiden werden, aus eigenem Interesse. Wie real das Szenario ist, zeigt die Forderung der Regierung in London, sehr zur Verärgerung Salmonds, ${ }^{62}$ die Notwendigkeit einer zweiten Volksabstimmung über die Ergebnisse der Verhandlungen bereits jetzt ins Gespräch zu bringen.

Unstrittig ist, dass das Verhandlungsergebnis durch Gesetze des englischen und schottischen Parlaments bestätigt werden müsste. ${ }^{63}$ Ob sich dabei die Erwartung erfüllt, dass es dabei nur noch um eine Formsache gehe, bliebt abzuwarten.

58 Vgl. Hazell, R.: Out of Court. Why Have the Courts Played No Role in Resolving Devolution Disputes in the United Kingdom?, in: Publius 37 (2007), 578 - 598.

59 Vgl. "Scottish independence is a UK issue", in: The Guardian online, 23.06.2011.

60 Vgl. "Scottish nationalists angry over double referendum hurdle", in: The Guardian online, 06.06.2011. .

61 Vgl. Scottish Government, a.a.O., 2007, 32; Keating, M., a.a.O., 2009, 84f; Andrew Black auf „Scottish independence referendum", in: BBC Scotland online, 29.05.2011.

62 Vgl. schon Scottish Government, a.a.O., 2007, 34.

63 Siehe Scottish Government, a.a.O., 2007, 20. 


\section{Die Lage nach englischem Verfassungsrecht}

Würden Whitehall und Westminster ein Referendum in Schottland zulassen, würde die britische Verfassung einer Unabhängigkeit Schottlands keine rechtlichen Hindernisse in den Weg stellen. Die Doktrin der absoluten Parlamentssouveränität erlaubt es dem britischen Parlament, jedes Gesetz zu erlassen, das ihm beliebt. ${ }^{64}$ Durch einfaches Gesetz könnte Schottland in die Unabhängigkeit entlassen werden, wie durch den India Act 1947 mit Indien verfahren wurde.

\section{Die Lage nach schottischer Verfassung(stheorie)}

Es liegt einige Ironie darin, dass der Fall sich nach schottischer Verfassung(stheorie) etwas komplizierter darstellt.

Die unermüdlichen Verfechter der schottischen Unabhängigkeit verwiesen in den sechziger Jahren des 20. Jahrhunderts darauf, dass die Union zwischen Schottland und England durch einen Vertrag (Treaty of Union) von 1706 zustande gekommen sei, dem gegenüber der Act of Union 1707 rechtlich zurücktreten müsse. Das werde unter anderem dadurch dokumentiert, dass der Act zahlreiche Formulierungen des Vertrages wörtlich übernommen habe. Wie es Verträgen zukomme, sei eine Änderung nur im gegenseitigen Einvernehmen möglich. ${ }^{65}$ Das würde bedeuten, dass die Unabhängigkeit Schottlands vertraglich vereinbart werden müsste. Allerdings findet sich auch die Auffassung, dass jeder Vertrag kündbar sei, auch jener über die Union von Schottland und England. ${ }^{66}$ Im Ergebnis handelt es sich hier um einen eher akademischen Streit, da solche Positionen für die Rechtspraxis in Großbritannien ohne Belang ist.

Einigkeit hingegen besteht darin, dass ein Referendum ein Gesetz des schottischen Parlaments zur Voraussetzung hat, unabhängig davon, ob es sich auf die Unabhängigkeit als solche oder auf die Ermächtigung der schottischen Regie-

64 Dicey, A.V.: Introduction to the Study of the Law of the Constitution, 8. Auflage, London, 1915, 37f; vgl. Schieren, S.: Großbritannien, a.a.O., 2011, 28ff; Keating, M., a.a.O., 2009, 82.

65 Vgl. Smith, T. B.: A Short Commentary on the Law of Scotland, Edinburgh, 1962, 57. Sollte der Treaty als ein internationaler Vertrag zu gelten haben, weil mit ihm die Inkorporierung Schottlands verbunden war (vgl. Dörr, O.: Die Inkorporation als Tatbestand der Staatensukzession, Berlin, 1995, 147, 195ff), wären ggf. Art. 54 und 56 Wiener Konvention über das Recht der Verträge zu beachten, die im Grundsatz die einseitige Kündigung ausschließen und die Möglichkeit einer Sezession daher an enge Bedingungen knüpfen. Vgl. Villiger, M. E.: Commentary on the 1969 Vienna Convention on the Laws of Treaties, Leiden/Boston, 2009, 681ff; 695ff.

66 Vgl. McLean, I./McMillan, A.: England and the Union Since 1707, in: Hazell, R. (Hg.): The English Question. Manchester/New York, 2006, 24 - 44, hier 27. 
rung, Verhandlungen mit Whitehall aufzunehmen, richtet. Solange die SNP über keine Mehrheit verfügte, gab es keinen verfassungsmäßigen Weg zu einem Referendum und damit zur Unabhängigkeit. ${ }^{67}$ Dieses Hindernis ist mit der Wahl vom Mai 2011 beseitigt worden.

\section{Zwischenergebnis und weiterführende Fragen}

Ungeachtet aller Unwägbarkeiten dürfte alles andere als eine einvernehmliche und nur so als rechtmäßig anzusehende Sezession auszuschließen sein. Konflikte über die neuen Grenzen wird es nicht geben, weil der territoriale Bestand von England und Schottland seit Jahrhunderten unverändert sowie (von Kleinigkeiten abgesehen) unstrittig ist und damit die Anwendung des uti possidetis-Prinzips möglich ist. ${ }^{68}$ Streitigkeiten über den Grenzverlauf zwischen den dann beiden neuen Staaten sollten die Verhandlungen nicht belasten. ${ }^{69}$

Damit wäre eine notwendige Bedingung für die grundsätzliche Zustimmungsbereitschaft der europäischen Partner zur Unabhängigkeit Schottlands und dessen Verbleib in der EU gegeben. Eine unrechtmäßige Sezession wäre für die Mitgliedstaaten inakzeptabel. Keinesfalls ist denkbar, dass ein abtrünniges Schottland umstandslos in der EU verbleiben bzw. in die EU aufgenommen werde würde.

Doch wie sieht es im Falle einer einvernehmlichen Spaltung aus? Haben die Partner keine andere Wahl, als Schottland dann als 28. Mitglied zu akzeptieren? Hat Schottland in diesem Fall einen rechtlich verbrieften Anspruch auf die fortgesetzte Mitgliedschaft in der EU? Woher könnte den Schotten dieses Recht zuwachsen? Welche Regelungen halten das europäische und das internationale Recht bereit, wenn es um die Frage der Fortgeltung internationaler Verträge geht? Wer kann welche Ansprüche erheben, wer muss welche Pflichten erfüllen?

\section{V. "Independence in Europe"?}

\section{Scottish independence und das Primärrecht der EU (Teil I)}

Zerfällt ein Staat oder trennt sich ein Teil von ihm ab, um einen eigenen Staat zu gründen und damit zu einem neuen Völkerrechtssubjekt zu werden, stellt sich die

67 Hazell, R.: Salmond, 2008, 18.

68 Vgl. Ott, M., a.a.O., 2008, 395ff; Fisch, J., a.a.O., 2010, 88ff; 105ff; Keating, M., a.a.O., 2009, 86.

69 Vgl. Tomuschat, Chr., a.a.O., 2006, 38. 
Frage, welcher Teil in die Rechte und Verpflichtungen aus internationalen Verträgen eintreten kann. Diese Frage hängt nicht allein von dem Willen der betroffenen Staaten ab. Bedeutsamer ist die Anerkennung durch die übrigen Vertragsstaaten. $^{70}$

Die europäischen Verträge enthalten keine rechtliche Regelung bezüglich der „,mitosis of a member state“ ${ }^{71}$ Die Suche nach Anhaltspunkten hinsichtlich des Vorgehens im Fall einer Sezession muss an anderer Stelle erfolgen. Wenden wir uns zunächst der Frage zu, ob die Geschichte der europäischen Integration Präzedenzfälle bereithält, die solche Anhaltspunkte liefern könnten.

Im Jahr 1957 wurde das Saarland als elftes Bundesland Teil der Bundesrepublik Deutschland. Bis dahin hatte es unter französischer Hoheit einen Sonderstatus inne. Obwohl mit diesem Akt erstens keine Änderung des Territoriums der Europäischen Gemeinschaft für Kohle und Staat verbunden war, Deutschland zweitens die Rechtsauffassung vertrat, dass das Saarland auch nach dem 8. Mai 1945 nicht aus dem deutschen Bundesstaats ausgeschieden war und drittens kein Mitgliedstaat Einwände gegen den Gebietsübergang hatte, wurde das im EGKS vorgesehene förmliche Vertragsänderungsverfahren durchgeführt, das zwei Jahre in Anspruch nahm. ${ }^{72}$

Im Jahr 1976 akzeptierten die Mitgliedstaaten, dass die winzige überseeische Besitzung und zu Frankreichs Hoheitsgebiet zählende Insel St. Pierre et Miquelon d'Outre Mer ohne förmlichen Antrag und aufwändiges Aufnahmeverfahren als Teil der EWG akzeptiert wurde. Die Gebietsänderung war zu unwesentlich, ${ }^{73}$ um sich mit dieser Frage auf andere Weise zu befassen. ${ }^{74}$

Der Abfall Algeriens von Frankreich 1962, der Antillen von den Niederlanden ebenfalls 1962, und der Austritt Grönlands ${ }^{75}$ aus der Gemeinschaft sind drei

70 Vgl. Schweisfurth, T., a.a.O., 1995, 87ff.

71 Lane, R: Scotland in Europe. An Independent Scotland and the European Community, in: Finnie, W./Himsworth, C. M. G./Walker, N. (Hg.): Edinburgh Essays in Public Law, Edinburgh, 1991, 143 157 , hier 144.

72 Ebd., 152.

73 Die Inselgruppe umfasst 242 qkm, auf denen gut 6000 Menschen leben.

74 Randelzhofer, A.: Deutsche Einheit und europäische Integration, in: Veröffentlichungen der Deutschen Staatsrechtslehrer 59 (1990), 101 - 122, hier 105ff; . Schröder, M.: Art. 236, in: von der Groeben, H./Thiesing, J./Ehlermann, C.-D. (Hg.): Kommentar zum EWG-Vertrag, Bd. 4, hg. von Christoph Bail, 4. Auflage, Baden-Baden,1990, 5645.

75 Harhoff, F.: Greenland's Withdrawal from the European Communities, in: Common Market Law Review 20 (1983), 13 - 33, hier 29; Johansen, O./Sørensen, C. L.: Grönlands Austritt aus der Europäischen Gemeinschaft, in: Europa-Archiv 38 (1983), 399 - 400. 
Fälle, die unterschiedliche gemeinschaftsrechtliche Implikationen hatten. Inwieweit die mit den Ereignissen verbundenen Änderungen vertragsrechtlich relevant waren oder nicht, wird in den Rechtswissenschaften unterschiedlich beurteilt. ${ }^{76}$ Weil Schottland aber nicht den Austritt aus der Union anstrebt, sondern im Gegenteil den ununterbrochenen Verbleib, sind die Beispiele nicht als Präzedenzfälle geeignet.

Der bedeutsamste Fall einer Änderung des Gemeinschaftsterritoriums sollte sich mit der Einigung Deutschlands 1990 ergeben. ${ }^{77}$ Die Umstände sind, soviel sei vorweggenommen, auch nicht geeignet, als Präzedenzfall für Schottland zu dienen. Dennoch ist eine genauere Betrachtung angezeigt, weil einige Aspekte von Belang sind, wenn es um eine politische Bewertung geht.

Die Ereignisse in der DDR brachten die Bundesregierung wie auch die erste frei gewählte Regierung der DDR im Verlauf des Jahres 1990 zu der Einschätzung, dass nur eine zügig in Aussicht gestellte Wiedervereinigung einen Zusammenbruch der Ordnung in der DDR und die damit verbundenen unabsehbaren Folgen verhindern könne. Als am 3. Oktober 1990 die deutsche Einigung vollzogen wurde, ging ein Staat unter. Er ging in einem anderen Staat auf, der sich entsprechend vergrößerte. Was beinahe unbemerkt ebenfalls geschah war, dass mit der Einigung auch eine substanzielle Erweiterung der EG vollzogen wurde, die der Erweiterung durch den Beitritt eines Staates nicht unähnlich war.

Der Beitritt eines Staats zur EU stellt allerdings ein aufwändiges und kompliziertes Verfahren dar (heute Art. 49 AEUV). Er setzt Einstimmigkeit im Rat und eine Vertragsänderung voraus, die ihrerseits durch alle Mitgliedstaaten ratifiziert werden muss. Seit dem Vertrag von Lissabon bedarf ein Beitritt zudem der mehrheitlichen Zustimmung des Europäischen Parlaments.

76 Schröder, M., a.a.O., 1990, 5641; Hilf, M.: Art. 240, in: von der Groeben, H./Thiesing, J./Ehlermann, C.-D. (Hg.): Kommentar zum EWG-Vertrag, Bd. 4, 4. Auflage, Baden-Baden, 1991, 5958; Schweitzer, M./Hummer, W.: Europarecht, 5. Auflage, Neuwied, 1996, 37; Kuyper, P. J.: The Community and State Secession in Respect of Treaties, in: Curtin, D./Heukels, T. (Hg.): Institutional Dynamics of European Integration, Bd. 2, Den Haag, 1994, 619 - 640, hier 623; Randelzhofer, A., a.a.O., 1990, 107;

77 Vgl. Giegerich, T.: The European Dimension of German Unification. East Germany's Integration into the European Communities, in: Zeitschrift für ausländisches öffentliches Recht und Völkerrecht 51 (1991), $384-450$. 
In der Literatur wurde kontrovers diskutiert, ${ }^{78}$ inwiefern ein formelles Beitrittsersuchen rechtlich geboten war. Die Erweiterung der Gemeinschaft um 17 Millionen Bewohner und $108.000 \mathrm{~km}^{2}$ durfte, im Unterschied zu St. Pierre et Miquelon d'Outre Mer, unzweifelhaft als eine substantielle Änderung angesehen werden. Durch den Vertrag von Maastricht 1993 erhielt das größere Deutschland zudem zusätzliche Sitze im Parlament und ein höheres Stimmgewicht im Rat, womit sich der Beitritt nachträglich auf die Balance in den Organen der EU auswirkte. Das lässt sich mit einiger Berechtigung als Verstoß gegen die Pflicht eines Mitgliedstaats deuten, Maßnahmen zu unterlassen, ,welche die Verwirklichung der Ziele dieses Vertrages gefährden könnten“ (Art. 5 Satz 3 EGV (Maastricht), heute Art. 4 Abs. 3 Satz 3 EUV). ${ }^{79}$

In den Turbulenzen der Jahre 1989/91 hatten sich allerdings die Bedingungen laufend verändert. Schließlich bestimmten die Ereignisse über den Ausgang des Prozesses, nicht gründliche politische Planung und sorgfältig verhandelte rechtliche Vereinbarungen. Ein Antrag auf Beitritt lag damals außerhalb der politischen Machbarkeit. Somit konnte sich Deutschland auf die rebus sic stantibus-Regel des Völkerrechts berufen. ${ }^{80}$

Angesichts dieser Umstände trafen die Partnerländer die stillschweigende Übereinkunft, ${ }^{81}$ die Frage des Beitritts der DDR im Zuge der Wiedervereinigung rechtlich nicht genauer zu betrachten und den Standpunkt einzunehmen, dass sich in Übereinstimmung mit Art. 29 Wiener Konvention über das Recht der

78 Verneinend Randelzhofer, A., a.a.O., 1990, 112; Grabitz, E./Bogdandy, A. von: Deutsche Einheit und europäische Integration, in: Neue Juristische Wochenschrift 43 (1990), 1073 - 1079, hier 1076; Schweitzer, M./Hummer, W., a.a.O., 35f; Schröder, M., a.a.O., 5645; Jacqué, J.P.: German Unification and the European Community, in: European Journal of International Law 2 (1991), 1 - 17, hier 4ff; Anker, A. $R$.: Wiedervereinigungsgebot, Europaintegrationsgebot. Eine Untersuchung anhand des Konflikts bei einer westeuropäischen Integration der Bundesrepublik Deutschland, München, 1991, $101 \mathrm{ff} ; 127 \mathrm{ff}$.

79 Aus heutiger Sicht erfüllt die Norm im Wesentlichen wohl eine andere Funktion; vgl. Kahl, W.: Art. 4 EUV, in: Callies, Chr./Ruffert, M. (Hg.): EUV/AEUV. Das Verfassungsrecht der Europäischen Union mit europäischer Grundrechtecharta, München, 2011, $101 \mathrm{ff}$.

80 Kuyper, P. J., a.a.O., 1994, 633, 639.

81 Im Einklang mit Art. 29 WKV. Vgl. Villiger, M. E., a.a.O., 393: „If there are territorial changes, the trety continues, in principle, to apply to the entire territority; different intentions would have to be renegotiated with, or at least be tacitly approved by, the other parties." Vgl. auch Timmermans, C. W.: German Unification and Community Law, in: Common Market Law Review 27 (1991), 437 - 449, hier 438; Jacqué, J. P., a.a.O., 11. 
Verträge $(\mathrm{WKV})^{82}$ die Gemeinschaftsgrenzen mit der Einigung einfach mit bewegt hätten. $^{83}$

Auf unseren Fall angewandt, würde die Doktrin der beweglichen Grenzen allerdings zu Ungunsten Schottlands wirken, weil sich die Grenze der Gemeinschaft nach der Sezession am Humber befände (oder bei Dover, sollte England nicht als Nachfolgestaat des Vereinigten Königreichs anerkannt werden), sofern Art. 29 WKV überhaupt als einschlägig anerkannt wird.

Liefert das EU-Recht somit keine Rechtsgrundlage für einen Anspruch Schottlands auf Sezession, hätte es bis zum Inkrafttreten des Lissaboner Vertrags am 1. Dezember 2009 möglicherweise sogar eine einvernehmliche Abspaltung erheblich erschwert. Müsste die Abspaltung formal als Austritt aus der EU bewertet werden, wofür viel spricht, hätte die EU darin bis zum Lissaboner Vertrag eine Vertragsverletzung sehen können, ${ }^{84}$ die einen solchen Schritt unrechtmäßig gemacht hätte, waren die Verträge doch ,,auf unbegrenzte Zeit““ (Art. 51 EUV und Art. 312 EGV jeweils i.d.F. von 2007) abgeschlossen worden. Mit dem Vertrag von Lissabon existiert jedoch seit dem 1. Dezember 2009 ein formelles Austrittrecht (Art. 50 AEUV). ${ }^{85}$ Damit verlor zumindest diese Frage an Relevanz.

\section{Scottish independence und das Recht der Staatennachfolge im Völker- recht}

Der Begriff „Staatennachfolge“ wird inzwischen weitgehend übereinstimmend als die „Ersetzung eines Staates durch einen anderen in der Wahrnehmung der internationalen Beziehungen eines Gebiets" verstanden. ${ }^{86}$ Wie sich dieser Prozess vollzieht, lässt eine ganze Reihe von Möglichkeiten zu.

Der Zerfall Russlands und Jugoslawiens führte zu unterschiedlichen Reaktionen seitens der UN. Die Resolution 777 des UN-Sicherheitsrats vom 19. September 1992 erkannte keinen der sechs neuen Staaten als Nachfolgestaat der Sozialisti-

82 Art. 29 WKV: ,Sofern keine abweichende Absicht aus dem Vertrag hervorgeht oder anderweitig festgestellt ist, bindet ein Vertrag jede Vertragspartei hinsichtlich ihres gesamten Hoheitsgebiets.“

83 Bulletin der Bundesregierung: EC Supplement 4/1990, S. 47ff.

84 Siehe Schieren, S.: Independence in Europe - Scotland's Choice?, in: Scottish Affairs 31 (2000), 111 127 , hier $124 \mathrm{f}$.

85 Callies, Chr.: Art. 50 EUV, in: Callies, Chr./Ruffert, M. (Hg.): EUV/AEUV. Das Verfassungsrecht der Europäischen Union mit europäischer Grundrechtecharta, München, 2011, 463 - 470, hier $463 \mathrm{ff}$.

86 Zimmermann, A.: Secession and State succession, in: Kohen, Marcelo G. (Hrsg.): Secession, Cambridge 2006, 208 - 230, hier 208. 
schen Bundesrepublik Jugoslawien an, auch nicht Serbien-Montenegro. Jeder Nachfolgestaat musste einen förmlichen Aufnahmeantrag zu den UN stellen. ${ }^{87}$

Im Gegensatz dazu wurde Russland als Nachfolgestaat der Sowjetunion anerkannt und trat in deren internationale Verträge ein. Das schloss auch ein, dass Russland den ständigen Sitz im Sicherheitsrat einnehmen konnte, der bis dahin von der UdSSR besetzt worden war, einschließlich des absoluten Veto-Rechts.

Über die internationalen Reaktionen auf eine Abspaltung Schottlands lassen sich nur Vermutungen anstellen. Die jugoslawische Variante wäre: Internationale Vereinbarungen verlieren sowohl für Schottland wie für England ihre Gültigkeit. Beide Staaten würden aus den internationalen Verträgen des Vereinigten Königreichs herausfallen. Diese Reaktion ist unwahrscheinlich, ${ }^{88}$ weil erstens die Sezession einseitig von Schottland gegen den Willen Englands durchgesetzt würde, und zweitens mit England-Wales (und Nordirland) ein neuer Staat entstünde, der hinsichtlich Bevölkerungsgröße und Fläche in erheblicher Weise mit dem Vorgängerstaat identisch wäre.

Daher ist die russische Variante die wahrscheinlichere: England wird als Nachfolgestaat anerkannt, für Schottland würden die internationalen Verträge ihre Geltung verlieren. Schottlands Wunsch nach Weiterbestand seiner Mitgliedschaft bliebe folglich in beiden Fällen unerfüllt.

Die Sicherheitsratsresolution 777 aus dem Jahr 1992 erfolgte allerdings vor InKraft-Treten der „Wiener Konvention über die Staatennachfolge in Hinblick auf Verträge“" ${ }^{89}$ Hat sich möglicherweise dadurch eine für Schottland günstigere Lage ergeben?

Auf den ersten Blick treten die Art. 34 und 35 WKV in den Vordergrund. Dort wird der Grundsatz geregelt, dass alle Nachfolgestaaten bei einer einvernehmlichen Sezession in die Verträge des Vorgängerstaats eintreten sollen. Mit der Einschränkung allerdings, dass damit die Ziele eines Vertrages nicht in Gefahr geraten dürfen.

Neben diesem Vorbehalt muss die insgesamt unverändert untergeordnete Bedeutung der Konvention in Rechnung gestellt werden, der eher konsultative als bin-

87 Rich, R., a.a.O., 54.

88 Lane (Lane, R., a.a.O., 146f) hat diese Variante als die folgerichtige bezeichnet, weil er den Austritt Schottlands als Zerfall und damit den Untergang des United Kingdom of Great Britain and Northern Ireland interpretiert. Der Aufsatz von Lane ist allerdings vor dem Zerfall der UdSSR entstanden.

89 UN Doc. A/CONF 80/16 Add. 2, 195. 
dende Wirkung zukommt. ${ }^{90}$ Das gilt besonders mit Blick auf die Rechtsnachfolge in Bezug auf internationale Verträge, für die die Konvention keine Anwendung findet. ${ }^{91}$ Gesetzespositivistisch gesehen zieht im Falle der Sezession der Vorgängerstaat seine Herrschaftsgewalt und seine Rechtsordnung und damit alle auf sie gegründeten Rechte von dem fraglichen Territorium zurück, so dass der neu entstandene Staat an eine „Stunde Null“ gelangt ist, jedenfalls mit Blick auf bestehende internationale Vereinbarungen. ${ }^{92}$

\section{Scottish independence und das Primärrecht der EU (Teil II)}

Weder das EU- noch das internationale Recht begründen mithin einen Anspruch auf eine Sezession Schottlands. Hier wird nun betrachtet, dass das EU-Recht sogar gewichtige Argumente dagegen liefert.

Spielen wir dazu das Szenario durch, dass Schottland nach einer Sezession Vollmitglied der EU bliebe. Den Grundsätzen der Verträge zufolge würde Schottland als souveräner Staat einen eigenen Sitz in der Kommission, im Europäischen Rat, im Rat, einen Richter beim Gerichtshof der Europäischen Union und eigene Abgeordnete im Europäischen Parlament erhalten, um nur die wichtigsten Organe zu berücksichtigen. Ebenfalls den Regeln des Vertrags zufolge würde Schottland mit seinen fünf Millionen Einwohnern Finnland vergleichbar in einem Teil dieser Organe weit überproportional zu seiner Bevölkerungsgröße vertreten sein, ohne dass England in gleicher Weise seine Vertretungsrechte einbüßen würde. Ein geteiltes „Großbritannien“ könnte folglich sein Stimmgewicht in den europäischen Organen elegant und signifikant erhöhen, obwohl dies erkennbar kein Motiv in der aktuellen Debatte darstellt.

Zum Zeitpunkt der ins Auge gefassten Unabhängigkeit 2016 wird im Rat ein neues Beschlussverfahren gelten. Beschlüsse benötigen die Mehrheit von 55\% der Mitglieder des Rats, die wiederum mindestens 15 (dann vielleicht 16?) Mitglieder ausmachen, die zugleich mindestens $65 \%$ der Bevölkerung repräsentie-

90 Siehe Schweisfurth, T., a.a.O., 1995, 203ff; Fastenrath, U.: Das Recht der Staatennachfolge, in: Neuhold, H./Simma, B. (Hg.): Neues europäisches Völkerrecht nach dem Ende des Ost-West-Konfliktes? Baden-Baden, 1996, $65-81$, hier 84ff.

91 Zimmermann, A., a.a.O., 220.

92 Fastenrath, U.: Das Recht der Staatensukzession, in: Das Recht der Staatensukzession. Berichte der Deutschen Gesellschaft für Völkerrecht, Bd. 35, Heidelberg, 1995, 9 - 44, hier 24. Bei der Staatensukzession gibt es eine Vielfalt rechtlicher Folgeprobleme zu beachten, die das Sozialrecht, Zivilrecht, Staatsangehörigkeitsrecht, die Streitkräfte, das Staatsvermögen, die Staatsarchive etc. betreffen. Ebd. 24ff; vgl. Schweisfurth, T., a.a.O., 1995, 82ff. 
ren müssen (Art. 16 Abs. 4 EUV). Es liegt auf der Hand, dass mit dem Anwachsen der Zahl der Mitglieder die Mehrheit immer schwerer zu erreichen sein wird. Das ließe sich wohl in dem Sinne interpretieren, dass eine Sezession Schottlands das Ziel „einer immer engeren Union der Völker Europas“ hinterfragen könnte. ${ }^{93}$ Zudem stünde der Vorwurf eines Verstoßes gegen Art. 4 Absatz 3 Satz EUV im Raum. Das ist keine reine Spekulation. Die schottische Regierung hat bei ihrer ersten Ankündigung 2007, auf die Unabhängigkeit hinarbeiten zu wollen, mitgeteilt, eine eigene Vertretung auf europäischer Ebene nachdrücklich im Interesse Schottlands nutzen $\mathrm{zu}$ wollen. ${ }^{94}$

Wegen dieser Schwierigkeiten erscheint es daher als naheliegender, dass die EU der Mitgliedschaft Schottlands nicht ohne Weiteres zustimmen würden. Flankierende politische Gründe verweisen sogar auf die Wahrscheinlichkeit der Blockade einer schottischen EU-Mitgliedschaft.

\section{Die politische Seite}

Wie bereits dargestellt, war es für die Wiedervereinigung enorm hilfreich, dass es unter den Mitgliedstaaten ein stillschweigendes Einverständnis gab, sich dieser nicht entgegenzustellen. Darüber hinaus konnte Deutschland auf die rebus sic stantibus-Regel im Völkerrecht verweisen.

Im Verhältnis dazu ist Schottlands rechtliche Position als schwach zu bezeichnen. Will es einen Wunsch nach Unabhängigkeit als Mitglied der EU verwirklichen, ist es auf die förmliche Zustimmung Englands und das Einvernehmen der anderen Mitgliedstaaten angewiesen. Auf beides kann Schottland allerdings nicht hoffen. Selbst der förmliche Beitritt nach Jahren der Verhandlungen ist bestenfalls denkbar, keinesfalls aber sichergestellt.

Die Reaktionen aus London sind unabhängig von der Parteizugehörigkeit einhellig. Ein Bruch der Union wird abgelehnt. Eine Sezession Schottlands würde zwar in Anerkennung des Willens des schottischen Volks im Einvernehmen mit England erfolgen, jedoch gegen dessen erklärten Wunsch und Willen. Unter diesen Umständen wäre es nur zu verständlich, wenn die englische Seite den dringenden Wunsch der Schotten auf Verbleib in der EU politisch instrumentalisieren würde. Eine diesbezügliche Erklärung Englands, sich in der EU dagegen aussprechen zu wollen, würde ein stilles Einvernehmen der Mitgliedstaaten, denen gegenüber

93 Vgl. schon Lane, R., a.a.O., $149 \mathrm{f}$.

94 Scottish Government, a.a.O., 2007, 23. 
England als Rechtsnachfolger Großbritanniens zählen würde, von vorneherein ausschließen und erheblichen Druck auf die schottischen Unterhändler erzeugen.

Vor diesem Hintergrund erscheint es naheliegend, dass Schottland zunächst aus der EU ausscheidet und einen Wiederaufnahmeantrag stellen muss. ${ }^{95}$ Dabei ist nicht allein ein möglicherweise auf Revanche zielendes England zu beachten, denn eine nüchtern kalkulierende Regierung in Whitehall müsste anerkennen, dass eine schottische EU-Mitgliedschaft auch im englischen Interesse läge. ${ }^{96}$ Jedoch haben die anderen Mitgliedstaaten das berechtigte Interesse der anderen Beitrittskandidaten zu achten, die bereits einen Antrag gestellt haben. Es ist daher nicht auszuschließen, dass sich Schottland hinter Bosnien-Herzegowina, Mazedonien und Kroatien einreihen muss.

Ein weiterer Grund spricht für ein solches Szenario: Die starke Zurückhaltung hinsichtlich der Frage der Anerkennung neuer Staaten in der Jugoslawienkrise ist unter anderen dadurch zu erklären, dass eine ganze Reihe europäischer Staaten nationale Minderheiten innerhalb ihrer Grenzen akkomodieren muss und durch ein unabhängiges Schottland auch ihre territoriale Integrität gefährdet sähen. ${ }^{97}$ In Spanien die Basken, in Frankreich die Korsen, Flamen und Wallonen in Belgien, und schließlich in Italien die Lega Nord, die die Trennung des reichen Nordens vom armen Mezzogiorno propagiert. Diese Staaten können kein Interesse daran haben, durch eine reibungslose Abspaltung Schottlands den Eindruck zu erwecken, die staatliche Unabhängigkeit könnte eine allgemein zustimmungsfähige und legitime Forderung sein. Schottland kann daher nicht mit Unterstützung der Mitgliedstaaten der EU rechnen - im Gegenteil.

Die ungenannte Prämisse bisher war, dass die Mitgliedstaaten bei einer Abspaltung Schottlands die russische Variante wählen würden. Das ist zwar wahrscheinlich, aber nicht gewiss. Aus politischen und nicht rechtlichen Gründen könnten die Mitgliedstaaten zumindest theoretisch auch die jugoslawische Variante wählen, um den politischen Druck auf England zu erhöhen, es gar nicht erst zu einer Abspaltung kommen zu lassen. Die Frage der Anerkennung von Staaten

95 Wie es die Nachfolgestaaten Jugoslawiens, der UdSSR und selbst die einvernehmlich getrennte Wege gehende Tschechoslowakei bezüglich der UN machen mussten. Russland ist hingegen in die ,Mitgliedschaftspositionen der UdSSR ,eingerückt““. Vgl. Schweisfurth, T., a.a.O., 1995, 113f; Zitat 117; Zimmermann, A., a.a.O., 220.

96 Keating, M., a.a.O., 2009, 90.

97 Vgl. Oeter, S.: Selbstbestimmungsrecht im Wandel, in: Zeitschrift für ausländisches öffentliches Recht und Völkerrecht 52 (1992), 751 - 780, hier 747f; Hazell, R., Salmond, a.a.O., 18; Keating, M., a.a.O., 2009, $90 \mathrm{f}$. 
bzw. der Staatennachfolge liegt schließlich in der Hand der anerkennenden Staaten, nicht der anzuerkennenden. ${ }^{98}$ Ebenso liegt die Frage der ununterbrochenen Mitgliedschaft Schottlands und Englands in der EU in den Händen der anderen Mitgliedstaaten. Die jugoslawische Variante würde bedeuten, dass England wie Schottland die Mitgliedschaft in der EU verlieren und sich für einen Wiedereintritt „hinten anstellen“ müsste. Das wäre eine Katastrophe für die wirtschaftlichen Interessen aller Teilgebiete des Vereinigten Königreichs, weswegen das Land ungeachtet aller ostentativen Europaskepsis und Europafeindschaft die Union bislang nicht verlassen hat. Es darf bezweifelt werden, dass England unter solchen Umständen einer Abspaltung Schottlands zustimmen wird.

\section{Schlussbetrachtung}

Durch ihren Sieg bei den Wahlen im Mai 2011 ist die Scottish National Party ihrem Ziel, die Unabhängigkeit Schottlands zu erreichen, ohne Zweifel ein großes Stück näher gekommen. Dabei beruft sie sich auf das vermeintliche Recht der Schotten, selbst und alleinig darüber zu bestimmen zu können, in einem souveränen Staat leben zu wollen.

Für diesen Anspruch gibt es aber keine rechtliche Grundlage, weder im Völkerrecht noch im europäischen Recht. Wie jedem Volk kommt den Schotten zwar das Selbstbestimmungsrecht zu, doch wird dieses Recht nicht dadurch verletzt, dass Schottland kein souveräner Staat ist. Vielmehr ist dem Selbstbestimmungsrecht ausreichend dadurch Genüge getan, dass die Schotten in einer rechtsstaatlichen Demokratie leben, in der sie zudem seit Beginn der devolution über eine Reihe wichtiger Fragen autonom entscheiden können. Ein Recht auf Sezession ist aus dem Selbstbestimmungsrecht jedenfalls nicht ableitbar. Das europäische Recht ändert diese Einschätzung allenfalls insofern, als es die Rechtsposition Schottlands noch schwächer erscheinen lässt.

Eine Sezession ist auf die Zustimmung nicht alleine Englands, sondern auch der anderen europäischen Staaten angewiesen. Das gilt besonders mit Blick auf die Mitgliedschaft eines souveränen Schottlands in der EU, die die schottische Regierung als eine Selbstverständlichkeit ansieht und mit der Formel „Independence in Europe" sogar als Argument dafür einsetzt, als kleiner Staat am Rande Europas nicht alleine zu stehen. Allerdings besitzt Schottland auch diesbezüglich keinen Rechtsanspruch, sondern ist auf die Zustimmung aller Mitgliedstaaten,

98 Vgl. Dugard, R./ Raič, D., a.a.O., 2006. 
einschließlich Englands, angewiesen, das aller Wahrscheinlichkeit nach als Nachfolgerin des Vereinigten Königreichs in dessen internationale Verträge eintreten wird. Diese Zustimmung darf jedoch aus politischen und rechtlichen Gründen nicht als selbstverständlich zugrunde gelegt werden. Im Gegenteil lässt sich die schwache schottische Rechtsposition sowohl durch die Regierung in London als auch durch die übrigen Mitgliedstaaten als Druckmittel einsetzten, um den Schotten den Wunsch nach Unabhängigkeit zu versagen.

Es spricht folglich Einiges dafür, dass sich der Weg zur Unabhängigkeit als steiniger erweist, als die Regierung in Edinburgh zurzeit glauben macht (und möglicherweise zur Kenntnis zu nehmen bereit ist). Sollte die Union bewahrt werden, dürfte dies weniger an deren Bindungskraft liegen, ${ }^{99}$ als an den Hürden des internationalen und vor allem des europäischen Rechts, die sich dem schottischen Unabhängigkeitsstreben in den Weg stellen.

99 So auch die Prognose von Hazell, R.: Conclusion. Where Will the Westminster Model End Up?, in: ders. (Hg.): Constitutional Futures Revisted. Britain's Constitution to 2020. London, 2008, 285 - 300, hier $289 \mathrm{f}$. 